Conclusions Successful development and implementation of preventive strategies against sports injuries in sport club settings continues. HA is currently planning new, modern strategies for implementation e.g. developing technological solutions (mobile and web applications) and webinars. Project is funded by Finnish Ministry of Education and Culture.

\section{THE INCIDENCE OF PHYSICAL CONTACTS IN YOUTH ICE HOCKEY ASSOCIATED WITH BODY CHECKING EXPERIENCE}

${ }^{1}$ Leticia Janzen, ${ }^{1}$ German Martinez, ${ }^{1}$ Maciek Krolikowski, ${ }^{1}$ Nicole Romanow, ${ }^{1}$ Luz PalaciosDerflingher, ${ }^{2}$ Luc Nadeau, ${ }^{1}$ Carolyn Emery. 'Sport Injury Prevention Research Centre, Faculty of Kinesiology, University of Calgary, Canada; ${ }^{2}$ Department of Physical Education, Faculty of Education, Laval University, Québec, Canada

\subsection{6/injuryprev-2016-042156.744}

Background Hockey Canada's 2013 body checking (BC) policy change was informed by evidence that $\mathrm{BC}$ leads to a $>3$-fold increased risk of injury compared with non-BC leagues. Video analyses found a reduction in high intensity physical contacts (PC) following this policy change. The association between BC experience and incidence of PCs has not been examined. As such, the incidence of intensity and types of PC were examined following the policy change in Pee Wee (PW) leagues (ages 11-12) with (Calgary) and without (Québec) BC experience.

Methods PW games were videotaped in Calgary $(\mathrm{N}=21$, with BC experience) and Québec City $(\mathrm{N}=20$, without BC experience), both non-BC leagues. Games were analysed using Dartfish with a validated observation system to quantify incidence of PC. Five levels of intensity (trunk contacts coded Level 1-5 intensity) and other types of PC (limb/head/stick). PC incidence rates per team-game and incidence rate ratios (IRR) (95\% CI) were estimated to compare games between two cohorts.

Results In total 4433 trunk contacts in Calgary and 2667 in Québec were recorded. Of the trunk contacts, 97.5\% (Calgary) and 95.7\% (Québec) were classified as low level PC. The incidence of total trunk contacts (number of contacts per team-game) was higher in Calgary than Québec (IRR $=1.58$, 95\% CI: $1.40-$ 1.79). There was no difference in high intensity contacts (Level 4 [IRR $=0.7295 \%$ CI: 0.48-1.07], Level 5 [IRR $=1.21$, 95\% CI: $0.57-2.56])$. The incidence of other PCs was lower in Calgary than Québec (IRR $=0.71,95 \%$ CI: 0.53-0.96).

Conclusion Following a policy change disallowing BC, PW players with experience $\mathrm{BC}$ had a greater incidence of total trunk contacts but not greater high intensity contacts than players without BC experience. Players with no experience had a higher incidence of other PCs. These results inform a greater understanding of mechanisms of contact in youth ice hockey that will in turn inform injury prevention and player development.

\section{AN ANALYSIS OF DENTAL TRAUMA AMONG YOUTH BASEBALL ATHLETES UTILISING SCHOOL INSURANCE BIG DATA IN JAPAN}

\footnotetext{
${ }^{1}$ Kinji Kusumoto, 1,2Yoshifumi Nishida, 1,2Koji Kitamura, 1,2Mikiko Oono, 1,2,3Tatsuhiro Yamanaka, ${ }^{4}$ Yutaka Sugimoto. 'National Institute of Advanced Industrial Science and Technology, Japan; ' Safe Kids Japan, Japan; ${ }^{3}$ Ryokuen Children's Clinic, Japan; ${ }^{4}$ Japan Sport Council, Japan
}

Background Japan Sport Council (JSC) collects more than $1,000,000$ injury data per year and provides medical benefits for injured students who got hurt under the supervision of school. From 2005 to 2014, JSC provided disability compensation for a total of 1,520 sports-related injuries. Baseball was the most common cause of injuries in youth sports, and 474 injuries occurred in extracurricular activities during the period. Kusumoto et al. ${ }^{1}$ found that eye trauma in baseball was the most common and that 196 cases occurred ( ${ }^{1}$ K. Kusumoto, Y. Nishida, K. Kitamura, M. Oono, T. Yamanaka, and Y. Sugimoto, "An analysis of ocular injuries among youth baseball athletes utilising school insurance big data in Japan," Safe Communities 2015 in Thailand, 2015). Kusumoto et al. also found that dental trauma was the second most common injuries in baseball. Therefore, in this study, we investigated dental injury among youth baseball athletes in Japan as a consecutive research.

Methods One hundred eighty-five injury cases were used for this research. First, we categorised these injuries by utilising JSC's injury severity grading chart defined by the Ministry of Health, Labour and Welfare in Japan. Next, types of injuries in each severity category were clarified based on dentists' diagnosis/ evaluation.

Results These injuries consisted of four groups by the injury grading chart. The groups range from severe to minor condition as follows; 1) 1 case (severity), 2) 7 cases, 3) 29 cases, 4) 188 cases (minor). The types of injuries were as follows; 93 teeth fracture, 54 dislocation/subluxation of teeth, 15 pulpitis, 13 alveolar bone fracture, 12 periodontitis and so on. Additionally, the most dental trauma occurred at the central incisors of upper teeth, more than 110 cases.

Conclusion Baseball-related dental trauma is the second highest common injuries among youth athletes in Japan. Based on the results, we propose some preventive methods; 1) wearing a helmet attached a full face guard, 2) managing their practice fields where usually multi-groups practice at the same schedule, and 3) educating coaches, athletes, and parents with potential risks of broken teeth in baseball.

\section{THE INCIDENCE OF BEHAVIOURS ASSOCIATED WITH BODY CHECKING EXPERIENCE AMONG YOUTH ICE HOCKEY PLAYERS}

${ }^{1}$ Thierry-Olivier Roy, ${ }^{2}$ Claude Goulet, ${ }^{2}$ Luc Nadeau, ${ }^{2}$ Kristine Fortier, ${ }^{3}$ Denis Hamel, ${ }^{4}$ Carolyn Emery. ${ }^{7}$ Department of Kinesiology, Faculty of Medicine, Laval University, Québec, Canada; '2Department of Physical Education, Faculty of Education, Laval University, Québec, Canada; ${ }^{3}$ Québec Public Health Institute, Québec, Canada; ${ }^{4}$ Sport Injury Prevention Research Centre, Faculty of Kinesiology, and Alberta Children's Hospital Research Institute, Departments of Paediatrics and Community Health Sciences, Cumming School of Medicine, University of Calgary, Canada

\subsection{6/injuryprev-2016-042156.746}

Background Participation and injury rates in youth ice hockey are high. Results of recent studies show that for 11 and 12 years old players the risk of injury is significantly higher in leagues where body checking (BC) is permitted compared to leagues where it is not. The objective of this research was to determine whether the incidence and types of body contact differ for 13 and 14 years old players in leagues where BC commenced at age 11 (Calgary) versus 13 and 14 years old players in leagues where BC was delayed until age 13 (Québec City).

Methods A cohort study was conducted in Québec City and Calgary. Sixteen games for Calgary and fifteen for Québec City were randomly selected and retrospectively analysed. Games were 